\title{
Preventing Glitches Circuit in Flash ADC
}

\author{
Jih-Fu Tu \\ Department Electronic Engineering, St. John’s University, Taiwan \\ tu@mail.sju.edu.tw
}

\begin{abstract}
The purpose of this paper is to design a prevented glitch circuit (PGC) to avoid the destroyed that is caused to glitch in the paralleling comparator Flash ADC. The advantages of this prevented glitch circuit are high-speed, lower power consumption, and size effective, furthermore, it also reduce the faults. We used the TSPC's D flip-flop to achieve this prevented glitch circuit where reduces and improves the glitches and faults, respectively. The PGC is simulated by the Tanner Pro. 13.0 with Generic0_25 $\mu \mathrm{m}$ techniques in $0.25-\mu \mathrm{m}$. Summarizing the features of this implemented circuit is lower power of $3.3 \mathrm{~V}$ at $333.3 \mathrm{MHz}$, higher area density is $89.6 \%$, and lower area size is $1221.16 \times 721.793 \mu \mathrm{m}^{2}$.
\end{abstract}

Keywords: Prevented glitch circuit, ADC, Tranconductance latched comparator.

\section{Introduction}

In the recent, many papers for ADC were published. In 2000's, [12] issued the Transconductance Latched Comparator circuit and based-on that to implement a switching ADC, but a fault caused by the glitch from decoder. In order to eliminate this glitch and remove it from the DAC, we exploited the TSPC (Technological systems for protective coatings) D-type flip-flop $[1,3,4,7,13]$ to improve the glitch problems in conventional ADC, to obtain a well waveform in output.

The focus of this paper was using the techniques of parallel comparator [6] to achieve a high-speed A-to-D transfer and lower noise interference, and then improve the glitches from Transconductance Latched Comparator. The ADC circuit derived from Terada et al. [12] that were shown in Figure 1, which is combined of FLASH A/D, sorter, and encoder.

This proposed prevented-glitch circuit aims at reducing the noise glitches to be generated from the output port of Transconductance Latched Comparator then to destroy the fine digital-signal. For an imprecise digital signal is delivered to the decoder that may cause the output signal jumping and fault.

Received: June $20^{\text {th }}, 2010 . \quad$ Accepted: April $10^{\text {th }}, 2011$ 


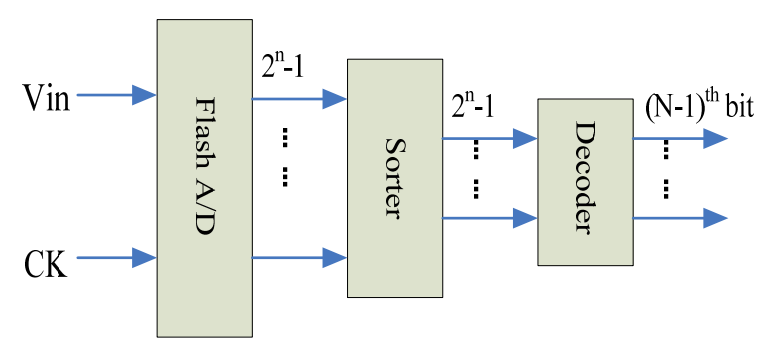

Figure 1. The ADC structure from Terada et al.

The contributions of this paper not only provide an accuracy digital signal but also lower glitch fault in the ADC's output port. We simulate this prevented glitch circuit using the Tanner Pro. 13.0 tool suit. The technology is Generic0_25 $\mu \mathrm{m}$ with $0.25-\mu \mathrm{m}$. The specifications of the implemented circuit as follows: 5-bit ADC, operation frequency is $333.3 \mathrm{MHz}$ at $3.3 \mathrm{~V}$, and the area is $1221.16 \times 721.793 \mu \mathrm{m}^{2}$.

The rent of this paper is organized as follows. Section 2 describes the relation works of DAC circuits, in the recent. The detail design process of this prevented glitch circuit is shown in Section 3. Section 4 presents the result of the improvement circuit and its fineness waveform. Section 5 shows the physical layout and remarks the conclusions.

\section{Previous Works}

In 2000's, Terada et al. integrated the Transconductance Latched Comparator $[5,8,12]$ into ADC that is compose of the dynamic circuit of FLASH A/D, and static circuit of SORTER and encoder. Because the front circuit needs timing signal of CLOCK. Though the dynamic has higher speed and less number of transistors than static circuit, it is difficult to design.

A parallel comparison Flash ADC circuit [6, 12] is shown in Figure 2 that is used to accomplish the Transconductance Latched Comparator, where had the following specifications: higher conversion speed from analogy to digital, and easily to implement a n-bit ADC only necessary to associate $\left(2^{n}-1\right)$ comparators, The $V_{\text {ref }}$ (Reference Value) is connected to the one of input pin of comparator through $2^{\mathrm{n}}$ serial resistors, and divide $\mathrm{V}_{\text {ref }}$ into equal $\left(\mathrm{V}_{\text {ref }} / 2^{\mathrm{n}}\right)$ 's value. The other pins of comparator are connected each other, moreover, an input signal $\mathrm{V}_{\text {in }}$, can feed through here. Comparing the magnitude of $V_{\text {in }}$ to $V_{\text {ref }}$, if $V_{\text {ref }}$ is lower than $V_{\text {in }}$ then an high-level signal is represented in the comparator's output, else the output is low-level.

The Flash ADC has higher-speed while analog converts to digital, but has more complexion circuit when archives an n-bit ADC need $2^{\mathrm{n}}-1$ comparators. Thus, this methodology doesn't suit to higher solution ADC. 


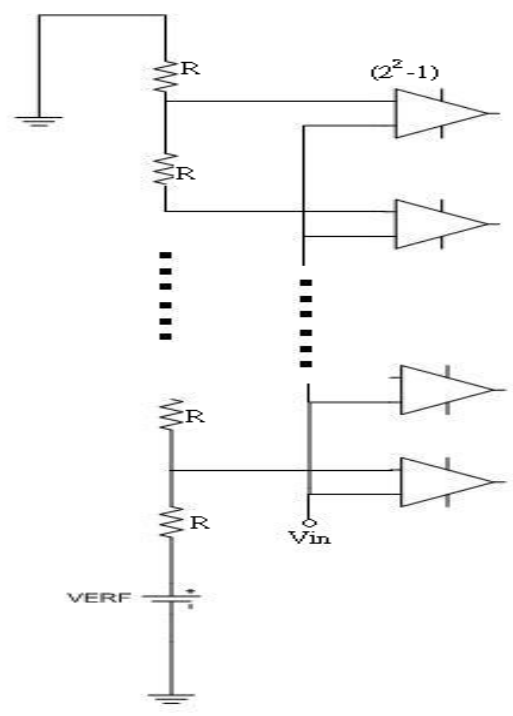

Figure 2. The circuit of Flash ADC.

\section{A. Transconductance Latched Comparator}

The Transconductance Latched Comparator is combined with four simply NOT gates $\left(\mathrm{NOT}_{1} \sim \mathrm{NOT}_{4}\right)$, six NMOS $\left(\mathrm{N}_{1} \sim \mathrm{N}_{6}\right)$ logic gates, and one PMOS $\left(\mathrm{P}_{1}\right)$. This Transconductance Latched Comparator is operated by three input signals such as clock (CK), $\mathrm{V}_{\mathrm{in}}$, and the reference voltage $\mathrm{V}_{\text {ref. }}$. It also has two output signals such as $\mathrm{V}_{\text {out }}$ and $\mathrm{V}_{\text {out }}{ }^{-}$, respectively.

The operation of Transconductance Latched Comparator is illustrated as Figure 3. When $V_{\text {in }}>V_{\text {ref }}$ and the clock is 0 , in this time, the circuit is controlled by $P_{1}$ switch. This circuit is trigged by the negative-edge.

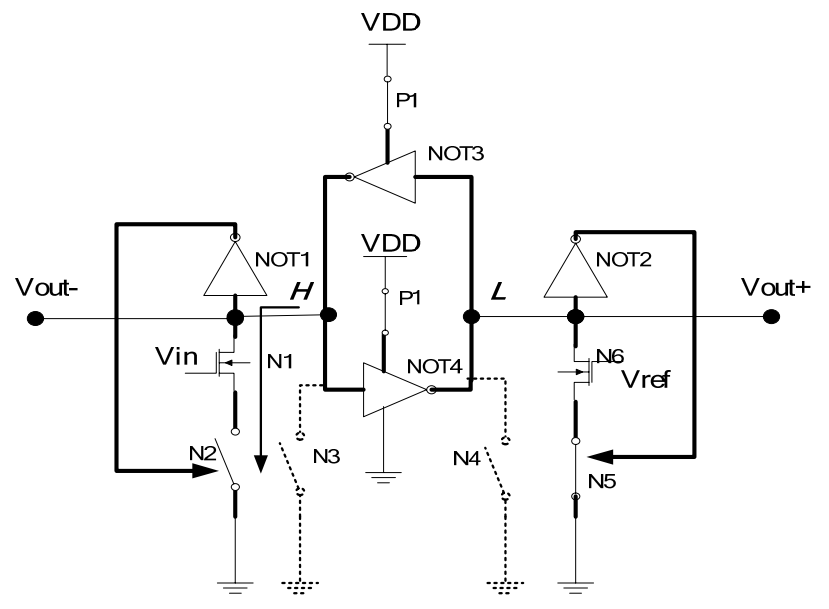

Figure 3. The operation of Transconductance Latched Comparator 
We found a resistor appearance in the output of Transconductance Latched Comparator that was cause of the transistors to due to the fault, which is shown in Figure 4 to be denoted red circles.

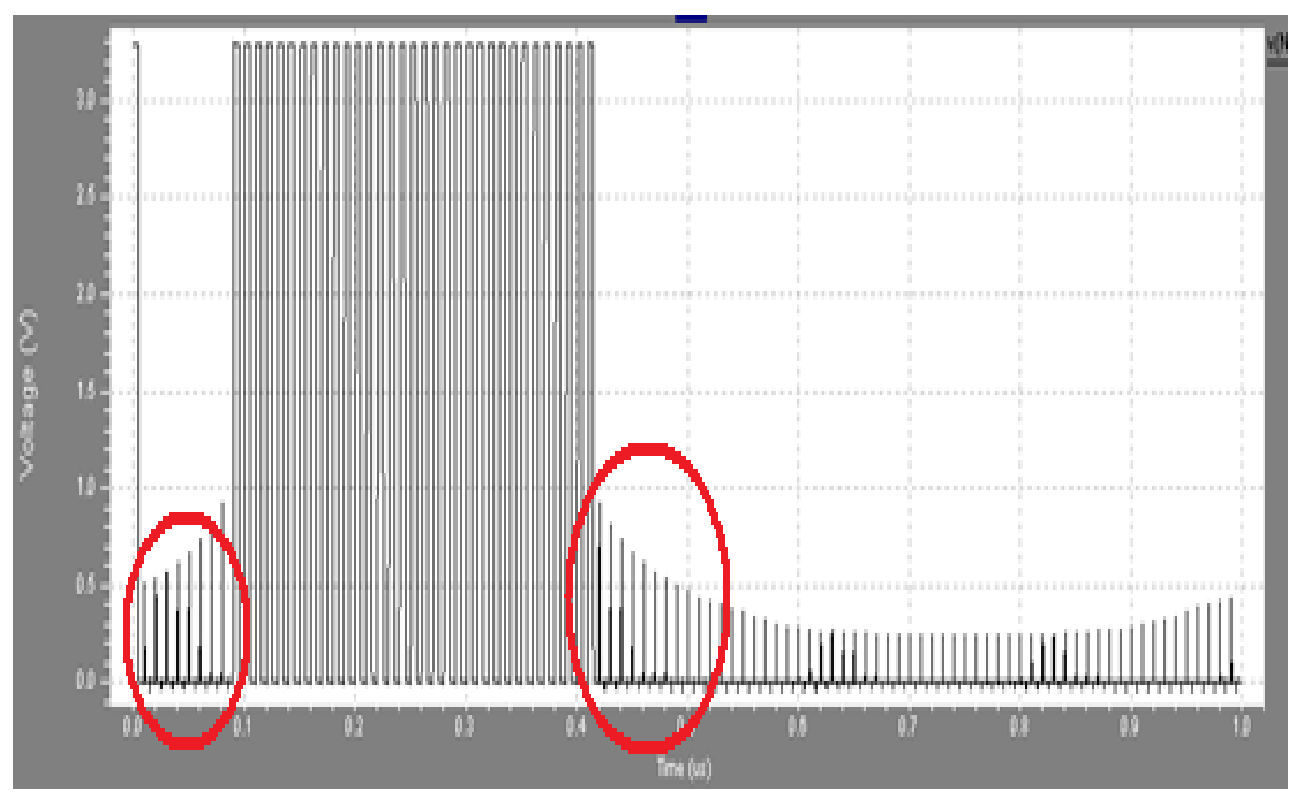

Figure 4. Resistor causes a fault.

The structure of flash A/D is shown in Figure 5, in where we insert a Butterfly Sorter to remove the faults to happen in output of ADC. SORTER in [12] was made up of n's butterfly sorter, which is interconnected AND gate and OR gate, to correct the faults to be generated by the resistor in the output of flash ADC.

The operation of Butterfly Sorter describes as follows, referring to the top-right of Figure 5, when $I_{1}$ and $I_{2}$ are fed to " 0 " and " 1 ", respectively. The output of $Q_{1}$ and $Q_{2}$ are associated to $I_{1}$ and $\mathrm{I}_{2}$, respectively. Further, it can correct the output wave and remove the fault cause for resistive. 


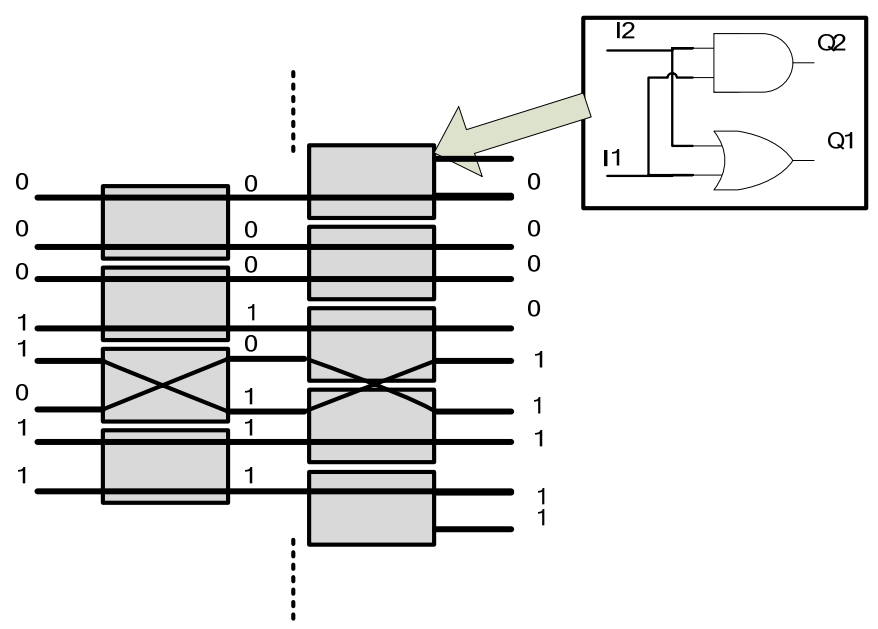

Figure 5. Butterfly Sorter [3].

\section{B. TSPC}

In this paper, we attempt to remove the glitch faults by the conventional TSPC DFF. The TSPC DFF is composed of three PMOSs and three NMOSs, and two input signals and one input output signal. The specifications of those two stages as follows. In the first stage, we make inverting and delay the input voltage $[2,5,10]$. On the other hand, in the second stage, we re-invert and delay the $\mathrm{V}_{\text {out1 }}\left(=\mathrm{V}_{\text {in2 } 2}\right)$. Why do re-invert in the second stage? The main reason is when the $\mathrm{CK}$ is low, the output of NMOS will make the output of PMOS to pre-charge up to $\mathrm{V}_{\mathrm{DD}}$, through the step-by-step method to obtain the necessary propagation, and the two-stage methodology circuit is shown in Figure 6. We use the charge-recharge flip action in CMOS to make the propagation and to restrain glitches.

Referring to Figure 6, the external second stage is accomplished to NAND gate. When in pre-charge phase, the output of $\mathrm{V}_{\text {out1 }}$ and $\mathrm{V}_{\text {out2 }}$ are pre-charged controlled by the PMOS_1 and PMOS_2, respectively. If a input signal of first stage occurs while CK is high-level, then the output of $\mathrm{V}_{\text {out1 }}$ and $\mathrm{V}_{\text {out2 }}$ are high-level, too. As the specification of two stages and double propagation that is used to implement the prevented glitch circuit (PGC). 


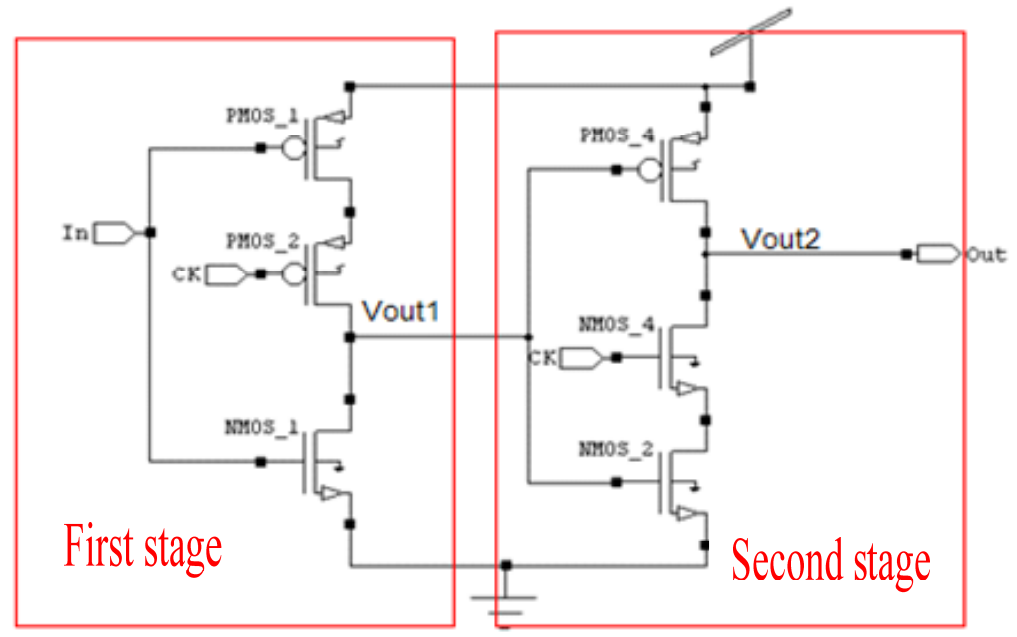

Figure 6. Two stages circuit.
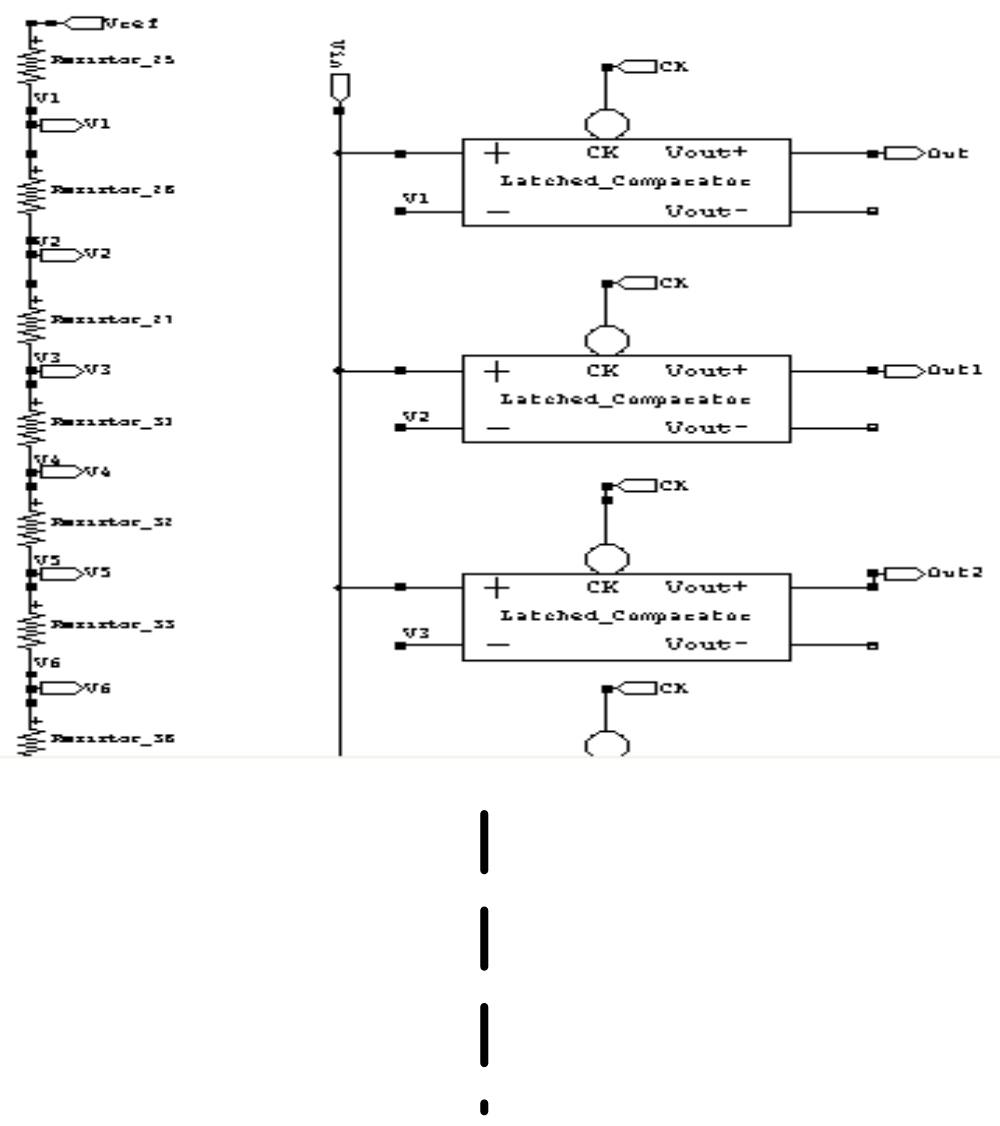

Figure 7. The structure of parallel ADC 


\section{PGC Design}

\section{A. Parallel ADC Structure}

The front-end of parallel of ADC is composed of two parts, the first part includes of $2^{\mathrm{n}}$ resistors, and the second part is combination of $2^{\mathrm{n}}-1$ comparators, as shown in Figure 7. The $\mathrm{V}_{\text {ref }}$ is fed to $2^{\mathrm{n}}$ resistors, and then divides into $2^{\mathrm{n}}$ equivalent $\mathrm{V}_{\text {ref }} / 2^{\mathrm{n}}$. Each of $\mathrm{V}_{\text {ref }} / 2^{\mathrm{n}}$ is connected to the corresponded comparator. When an analog signal occurs in ADC, it will be compared to $\mathrm{V}_{\text {ref }}$, immediately. If the input signal is larger than $\mathrm{V}_{\text {ref }}$ then the analog signal will be delivered to the next stage, else it will be blocked.

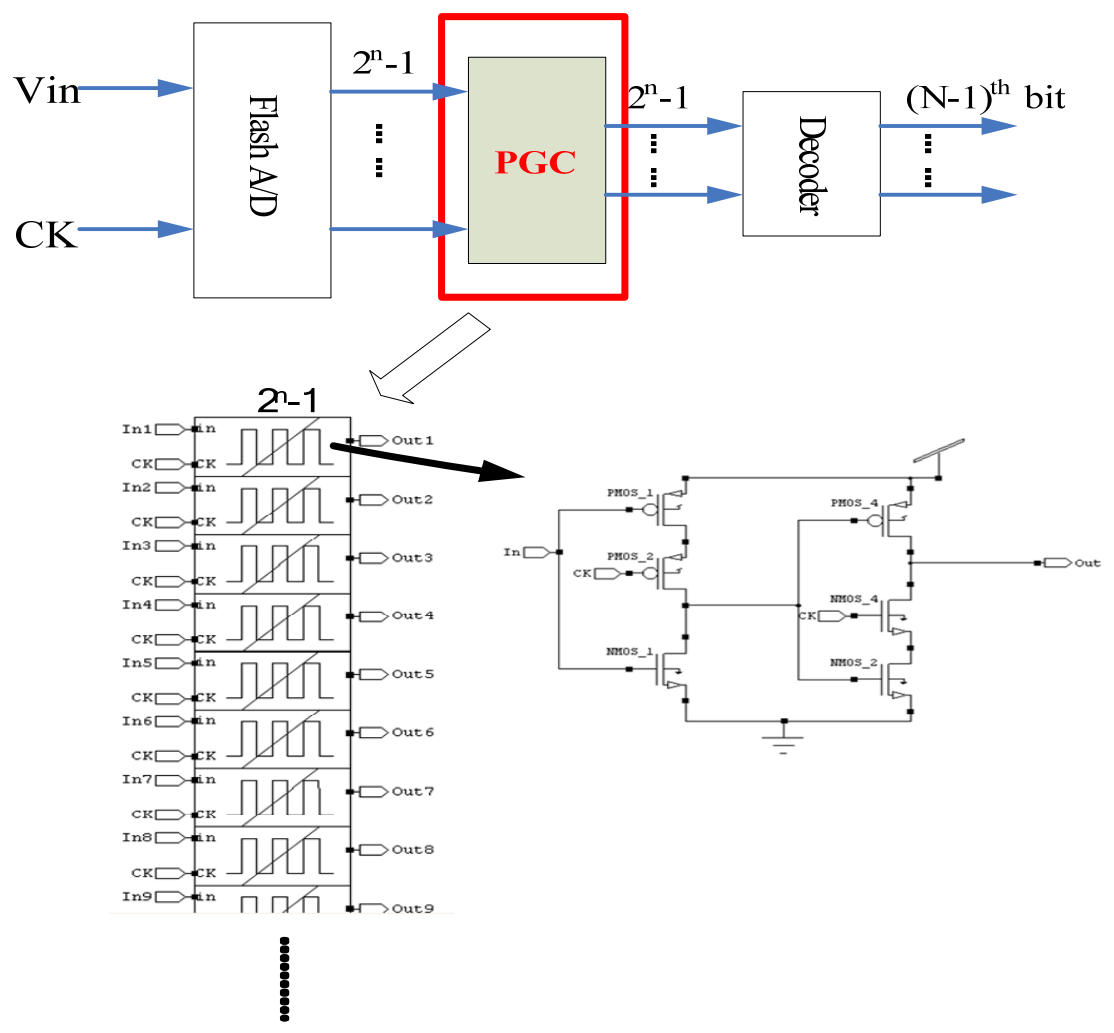

Figure 8. A PGC block with $2^{\text {n }}-1$ sub-circuits

\section{B. Prevented Glitch Circuit (PGC)}

We exploit the two-stage method, which is described Figure 6, to achieve the circuit of PGC that is shown in Figure 8. The PGC block is composed of $2^{n}-1$ sub-circuits. Where $n$ is the bit number of ADC, for instance, a 5-bit ADC needs $2^{5}-1=31$ two-stage circuits are embedded into the PGC block. That is, we must prepare 31 Parallel ADCs (as shown in Figure 7) to match PGC sub-circuits. 


\section{Encoder Circuit}

The encoder is implemented to the traditional logic-gate, such as AND, OR, and NOT [8, 9 , 11]. For instance, a 5-bit ADC of this work has 31 input signals and 5 output signals.

\section{The results and Analysis}

The physical of this proposed 5-bit ADC is shown Figure 9. It works in $333.33 \mathrm{MHz}$, the operation voltage is $3.3 \mathrm{~V}$, in addition to three input signals (analog input $\mathrm{V}_{\text {in }}, \mathrm{V}_{\text {ref }}$, and $\mathrm{CK}$ ), five output pins denote 5-bit such as $\mathrm{O}_{1}, \mathrm{O}_{2}, \mathrm{O}_{3}, \mathrm{O}_{4}$, and $\mathrm{O}_{5}$, respectively.

\section{A. The Output in PGC}

We use the butterfly characteristic of charge/re-charge to create the propagation, and then remove the glitches in clock. The charge phase occurs in the $1^{\text {st }}$ stage and re-charge happens in the $2^{\text {nd }}$ stage, both outputs are $\mathrm{V}_{\text {out1 }}$ and $\mathrm{V}_{\text {out } 2}$, respectively. The wave of $1^{\text {st }}$ output and $2^{\text {nd }}$ input are shown in Figure 10.

We reuse the function of delay causes for reversing. Consequently, in the output port may generate higher density to gain the effect on removing glitch that result is shown in Figure11. The relation of outputs of each stage in PGC is shown in Figure 12.

We probe the output wave of flash ADC in PGC and the CK to find a delay time occurring in this circuit, in where the delay time is one clock cycle and to show in Figure 13.

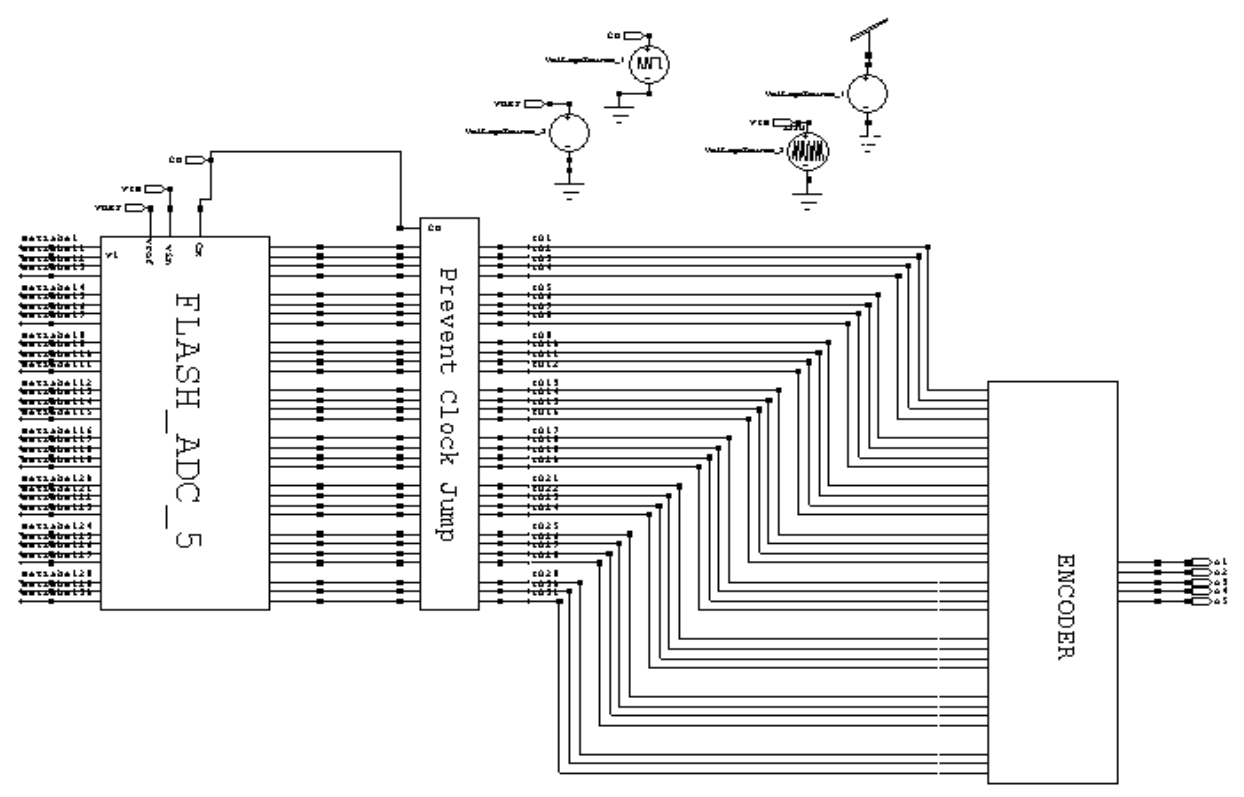

Figure 9. The schematic of 5-bit ADC in this work. 
Preventing Glitches Circuit in Flash ADC

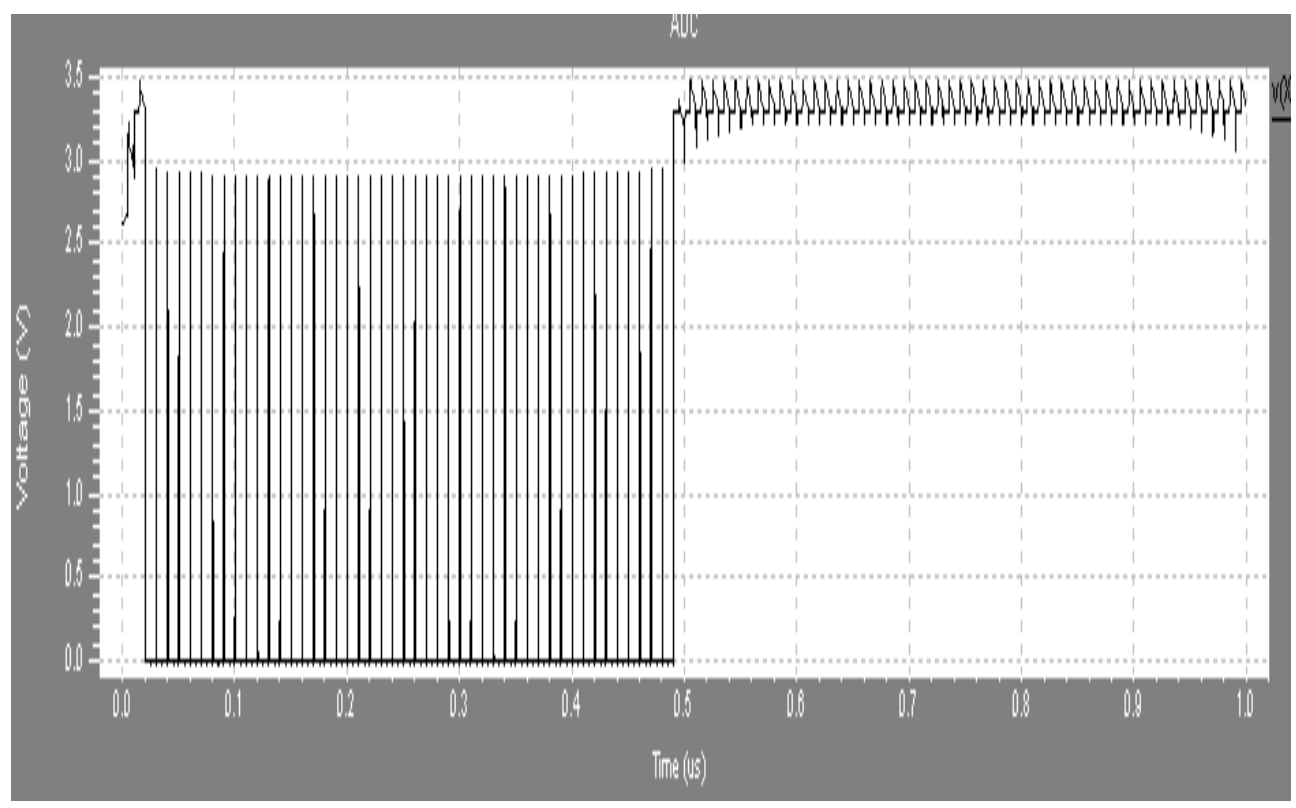

Figure 10. The wave in $1^{\text {st }}$ and $2^{\text {nd }}$ stage of PGC.

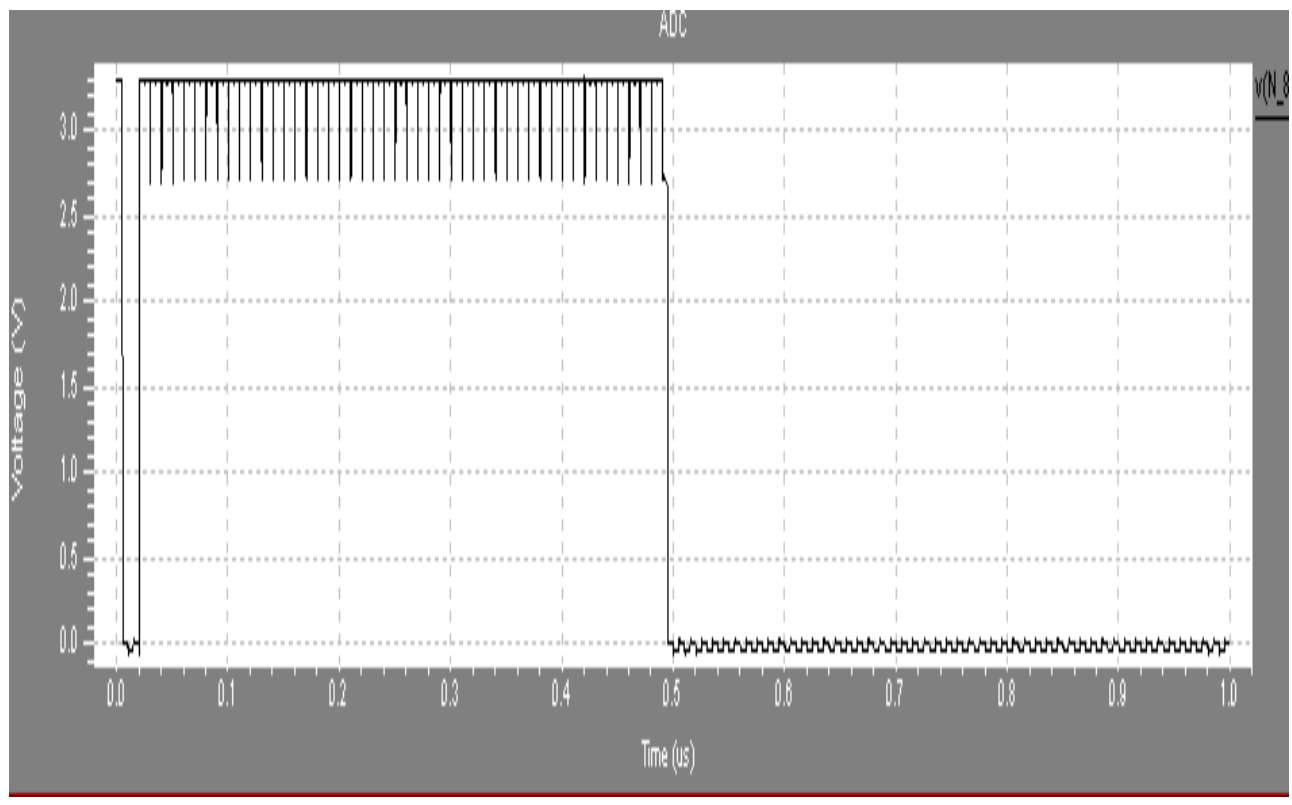

Figure 11. The output of PGC in the $2^{\text {nd }}$ stage. 


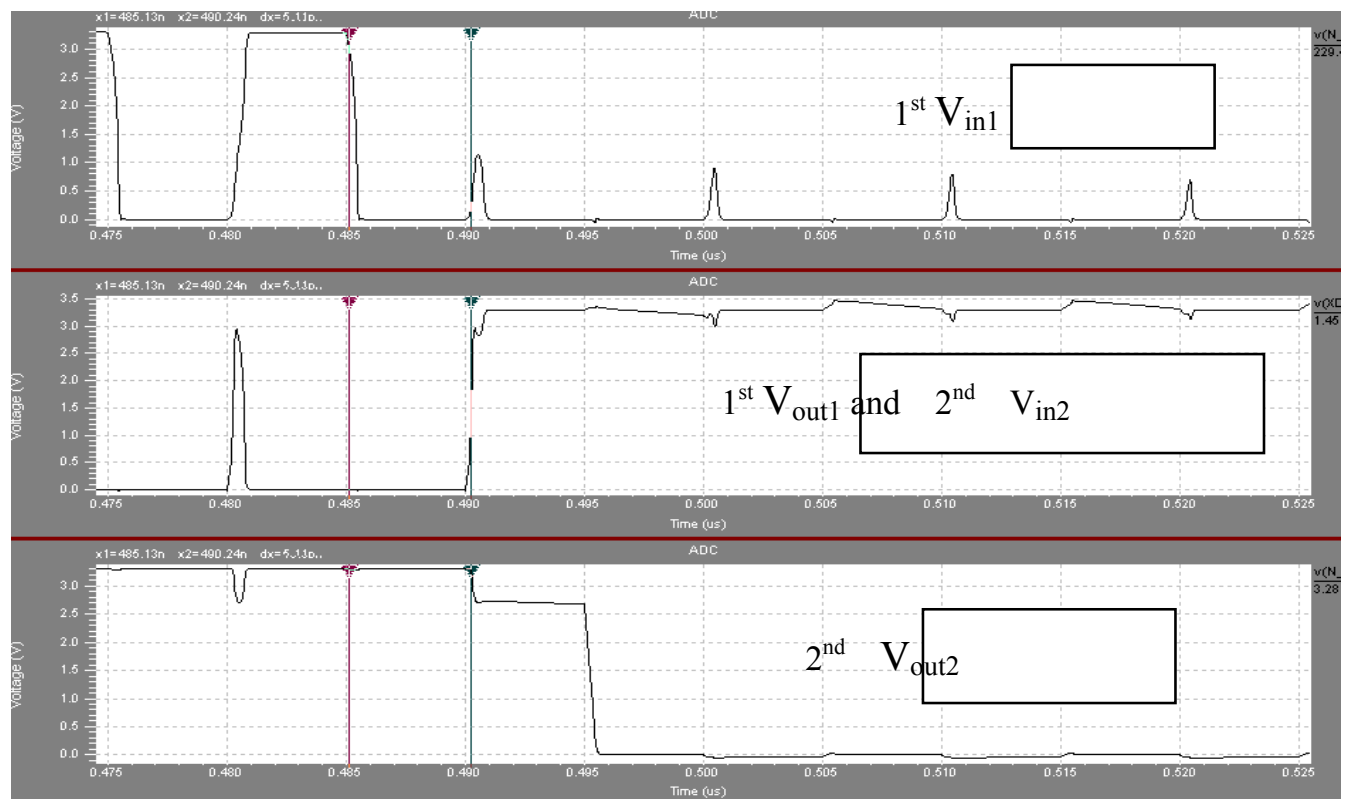

Figure 12. The output in PGC's each stage.

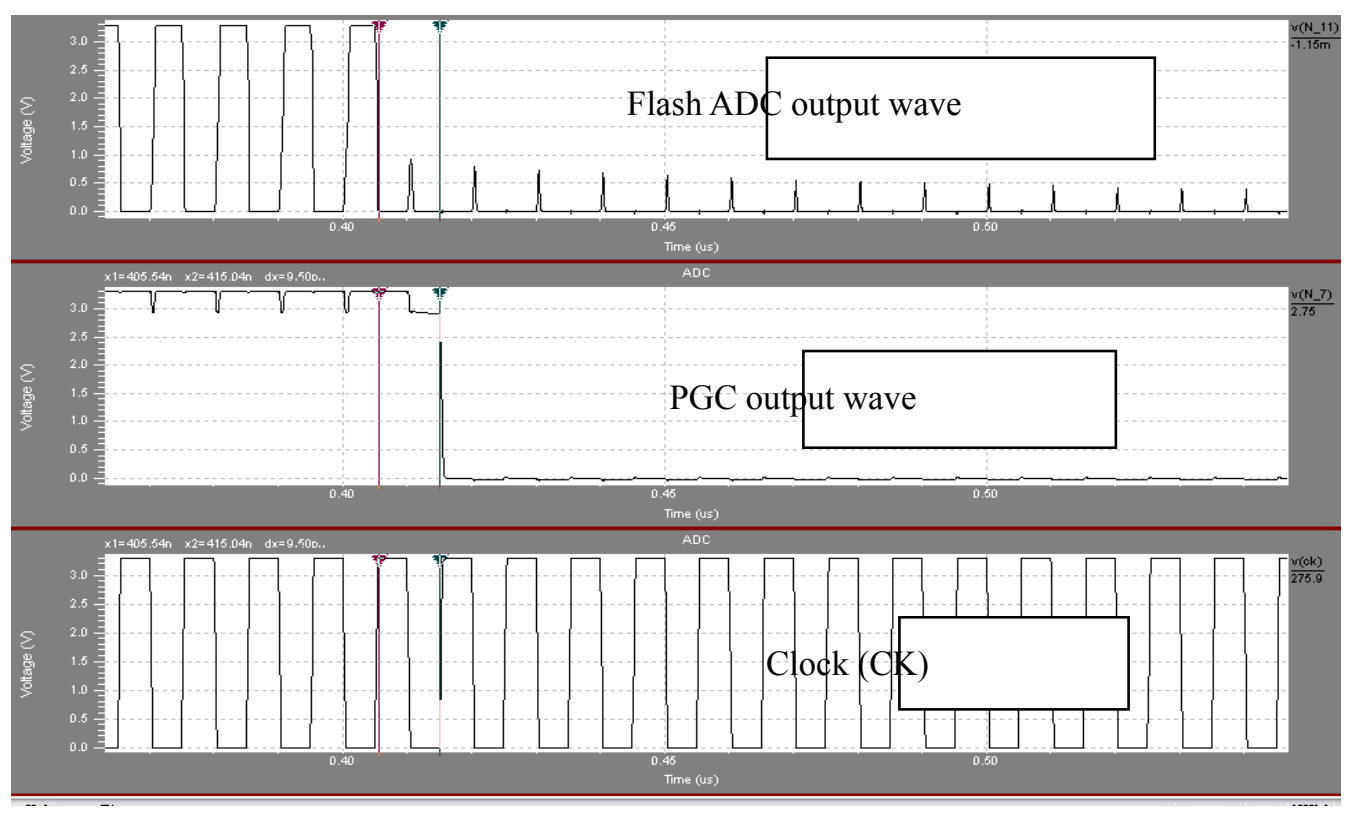

Figure 13. The output wave in flash-A/D and PGC.

An input analog signal is processed by the achieved PGC's circuit. A stable and fine wave occurs in the output of PGC that is shown in Figure 14. We observe the output wave not find enormous glitch in its output. 


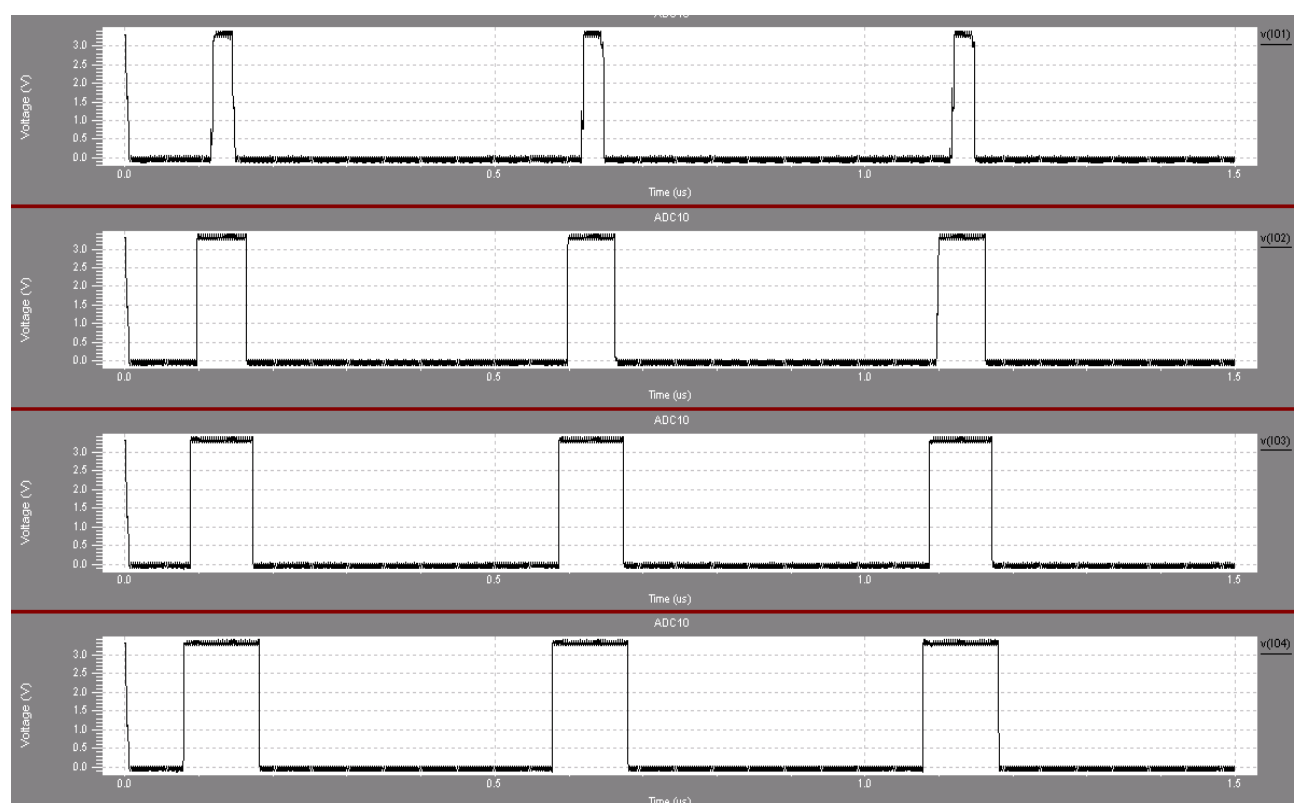

Figure 14. The fine wave in PGC's output.

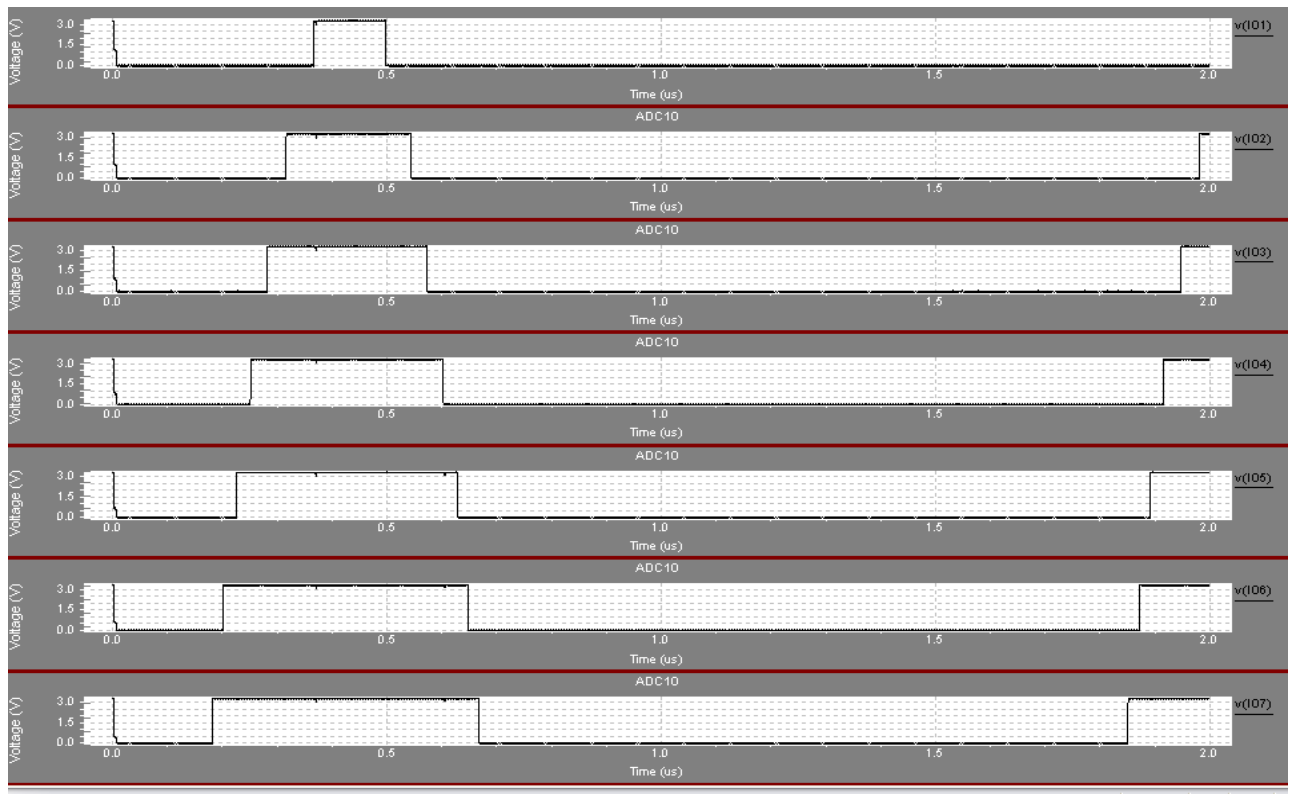

Figure 15. The output wave of ADC in lower order (1-7 digital-step). 


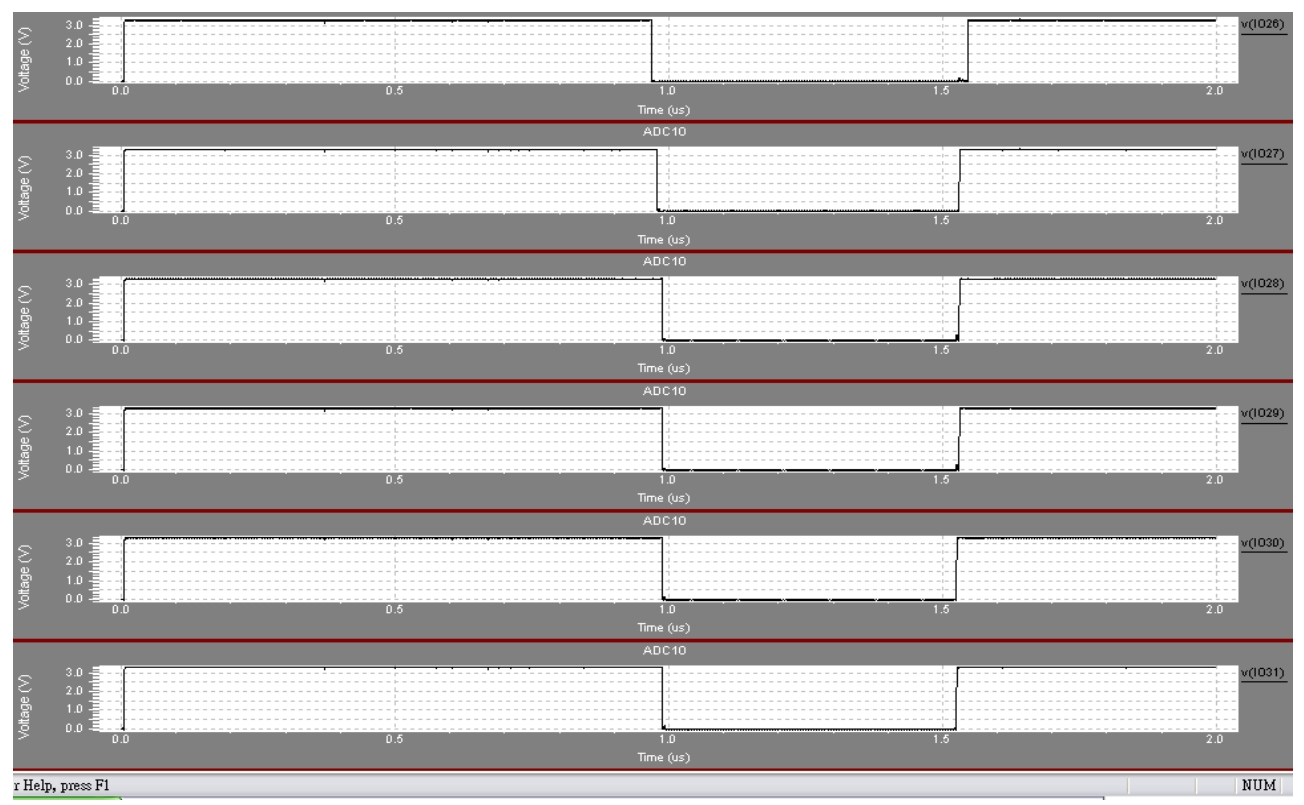

Figure 16. The output wave of ADC in higher order (26-31 digital-step).

\section{B. The Output-wave of 5-bit ADC}

For a 5-bit ADC have 31 digitizing output signals. The output wave of 1-7 lower order and 26-31 higher order are shown in Figure 15 and Figure 16, respectively. We observe the output wave finding it very well and fine, and not any glitch jump occurrence.

We also make this improved PGC circuit operating in $166.67 \mathrm{MHz}$ and $333.3 \mathrm{MHz}$, the output wave are shown in Figure 17 and Figure 18, respectively.

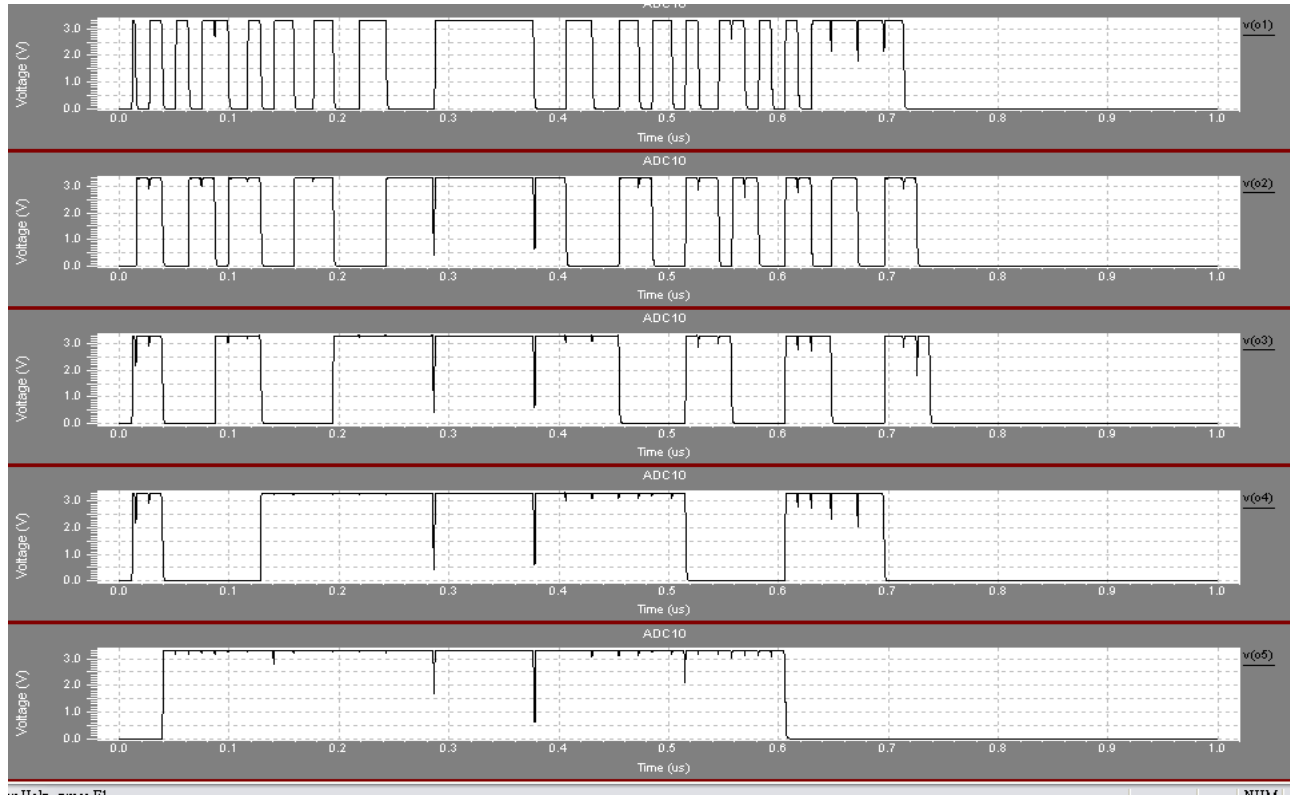

Figure 17. The output of this work at $166.67 \mathrm{MHz}$. 


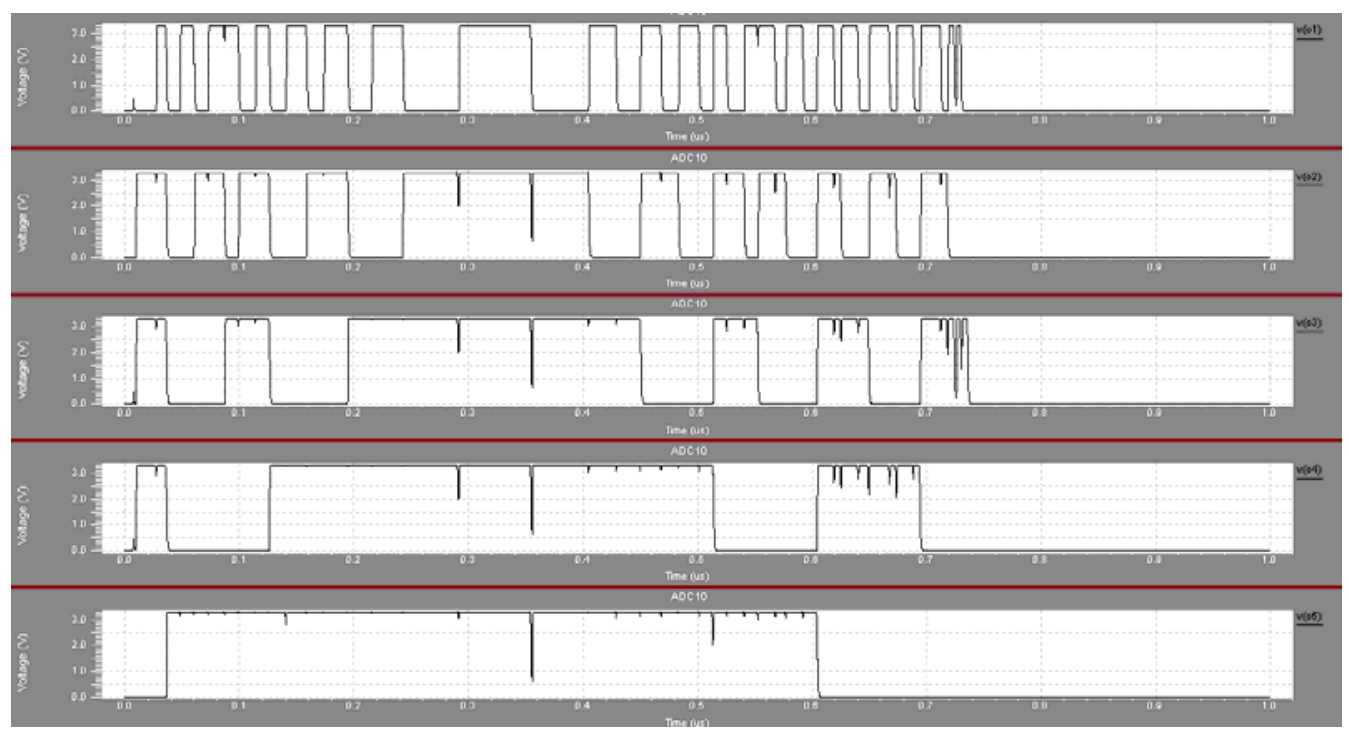

Figure 18. The output of 5-bit ADC for this work at 333.3MHz

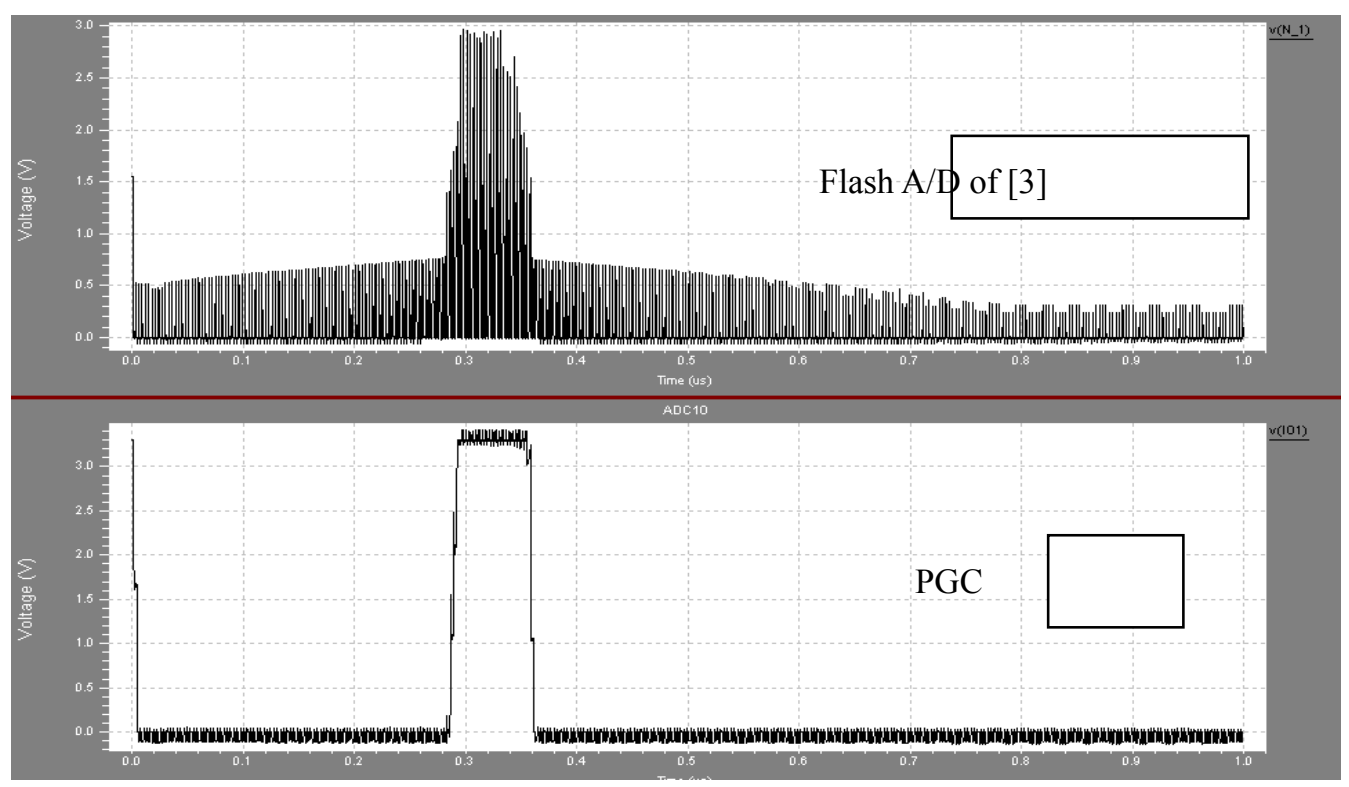

Figure 19. The output of [3] and PGC at 333.3MHz.

\section{Performance Analysis}

In this section, we compared the output wave of this implemented PGC to [12], both two are operated in $333.3 \mathrm{MHz}$ and $166.67 \mathrm{MHz}$. The output wave is shown in Figure 19 and Figure 20. We find that this improved PGC has higher stability and more fineness than [12]. As the result, that proves the improved $\mathrm{ADC}$ circuit not only reducing the area of transistors, but also 
Jih-Fu Tu

removing the glitch faults.

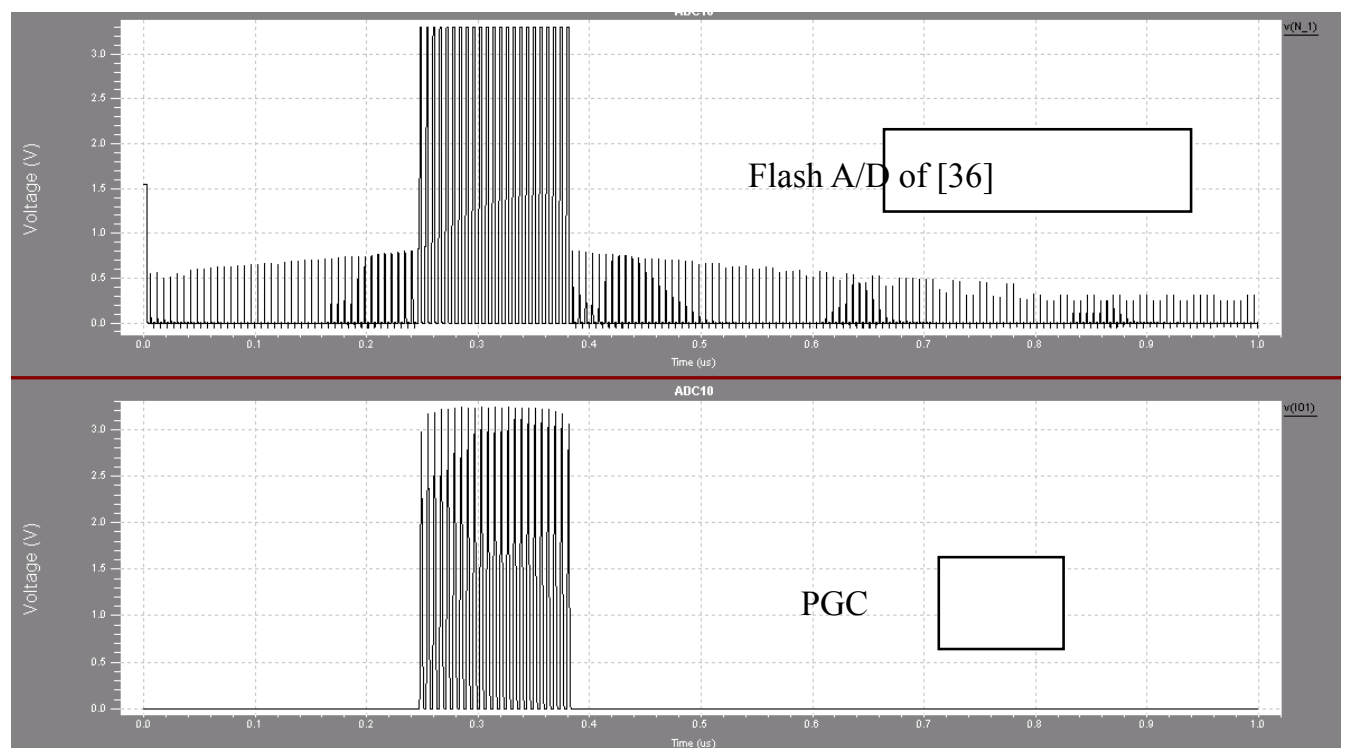

Figure 20. The output of [3] and PGC at 166.67MHz.

In this paper, we also simulated this proposed circuit using Tanner Pro Tools 13.0, and the technology was Generic0_25 $\mu \mathrm{m} 0.25-\mu \mathrm{m}$. Figure 21 shows the layout of the whole 5-bit DAC in our work, and its area is $1221.16 \times 721.793 \mu \mathrm{m}^{2}$, only.

We compared our work with [3], we replaced the SORTER by the PGC to reach the goal for preventing glitches. The original structure of the Butterfly Sorter is composed of AND2 and OR2. In other words, we need 6 CMOS transistors to construct the AND2 and OR2. If we want to implement a 5-bit ADC, in which must support 31 Butterfly Sorter, i.e. the numbers of CMOS transistors is $186(6 \times 31)$. But, we only need 98 CMOS transistors to construct the PGC. As the result, we can reduce one half area that one half the numbers of CMOS transistors to the traditional structures.

Through simulating, we analyze the output wave of PGC to find that is more stable and fine than [3]. We show that the PGC can implement the goal to reduce glitch and faults.

\section{Conclusions}

A 5-bit DAC with the characteristic of glitch prevent $\mathrm{s}$ is proposed to achieve high efficiency operation. This proposed circuit is architected to Tanner Pro. Tools 13.0, and the technology is Generic $025 \mu \mathrm{m} 0.25-\mu \mathrm{m}$. And then the die area of this 5-bit DAC is $1221.16 \times 721.793 \mu \mathrm{m} 2$. For the power consumption is $3.3 \mathrm{~V}$ when the operation frequency is 
333.3MHz. As for the cost reduction, we need one half the numbers of CMOS transistors to the traditional structures while it is configured and the density is $89.6 \%$. The ADC is popularity application to many system, thus, we have much room to improve the structure of higher-order ADC with hybrid configuration using parallel model or flash model, etc.

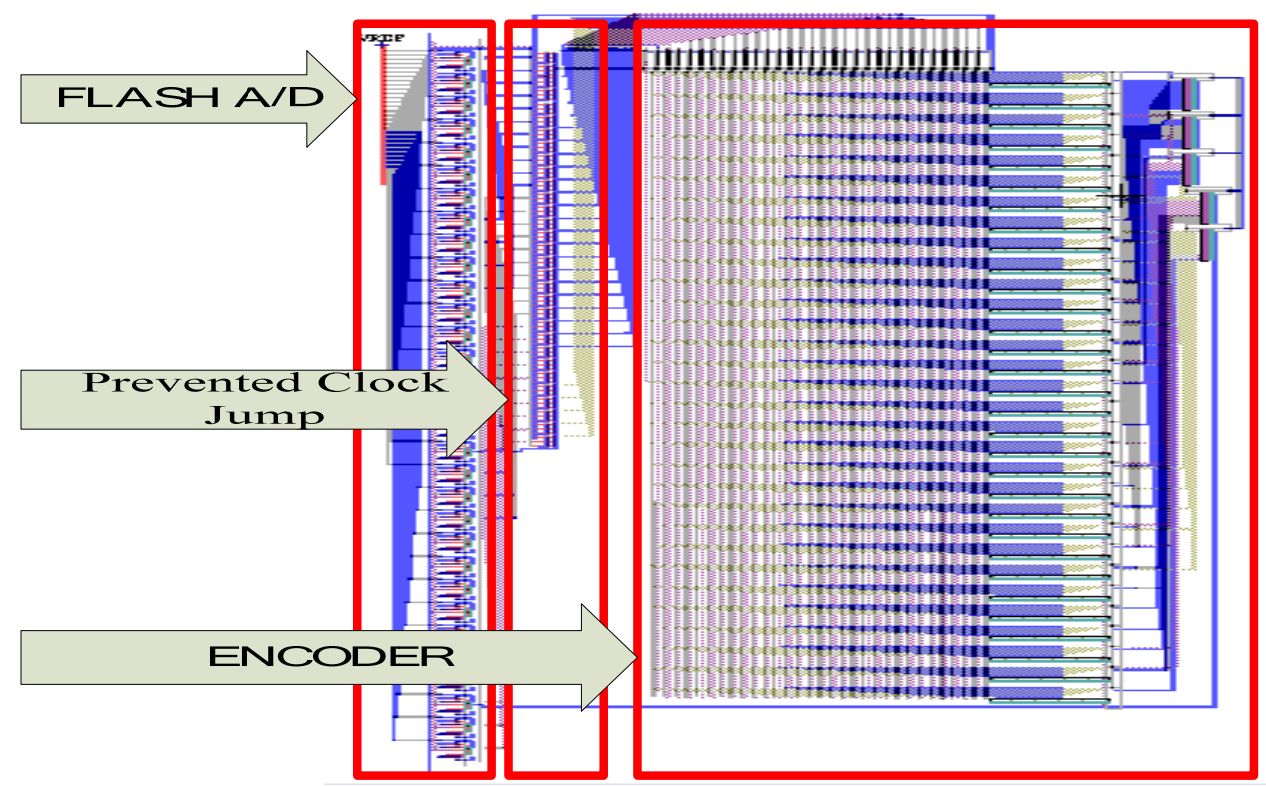

Figure 21. The layout of 5-bit DAC of our work.

\section{References}

[1] Antaki, S. Patenaude, L. Trognon, and Y. Savaria, "A Study on Split-Output TSPC CMOS Circuits," Proceedings of 1997 IEEE International Symposium on Circuits and Systems, ISCAS '97, Volume 3, 9-12 June. 1997, pp.1892 - 1895.

[2] S. Arlt, G. Scarbata, S. fitter, and C. Wisser, "Telescopic Layout Cells for Analog CMOS Circuits," Proceedings Euro ASIC '92, 1-5 June. 1992, pp. 139 - 143.

[3] Kuo-Hsing Cheng, and Yung-Chong Huang, "The non-full voltage swing TSPC (NSTSPC) logic design," Proceedings of the Second IEEE Asia Pacific Conference on ASICs, AP-ASIC 2000, 28-30 Aug. 2000, pp.37 - 40.

[4] Shun-Wen Cheng, "64-bit Pipeline Conditional Carry Adder with MTCMOS TSPC Logic," 50th Midwest Symposium on Circuits and Systems, MWSCAS 2007, 5-8 Aug. 2007 , pp.879-882.

[5] K. Gulati, and H.-S. Lee, “A \pm 2.45 V-swing CMOS telescopic operational amplifier," $45^{\text {th }}$ IEEE International Digital Object Identifier Solid-State Circuits Conference, 1998, 5-7 
Feb. 1998, pp.324 - 325, 456.

[6] T. Y. Hsu, and Z. M. Lin, “A 12-Bit Direct Level-Signal Transition Based Pipelined Analog-to-Digital Converter," IEEE Conference on Digital Object Identifier Electron Devices and Solid-State Circuits, 2007, 20-22 Dec. 2007, pp.881 - 884.

[7] Seungsoo Kim, and Hyunchol Shin, "Investigation of forward body bias effects on TSPC RF frequency dividers in $0.18 \mu \mathrm{m}$ CMOS," International SoC Design Conference, ISOCC '08, Volume 01, 24-25 Nov. 2008, pp.I-410 - I-413.

[8] Y. M. Lin, B. Kim, and P. R. Gray, "A 13-b 2.5MHz Self-Calibrated Pipelined A/D Converter in 3-um CMOS," IEEE, JSSC, Vol. 26, April. 1991, pp. 628-636.

[9] Y. Matsuya, and J. Yamada, “1 V Power Supply, Low-Power Consumption A/D Conversion Technique with Swing-Suppression Noise Shaping," IEEE Journal on Solid State Circuits, 1994; 29:1524.

[10] R. Ockey, and M. Syrzycki, "Optimization of a latched comparator for high-speed analog-to-digital converter," IEEE Canadian Conference on Electrical and Computer Engineering, Vol. 1, 1999, pp. 403-408.

[11] Behzad Razavi, "Design of Analog CMOS Integrated Circuits," McGraw-Hill, 2001.

[12] J. Terada, Y. Matsuya, F. Morisawa, and Y. Kado, "8-mW, 1-V, 100-MSPS, 6-BIT A/D CONVERTER USING A TRANSCONDUCTANCE LATCHED COMPARATOR," Proceedings of the Second IEEE Conference on AP-ASIC, Aug. 28-30, 2000, pp.53 - 56.

[13] Chua-Chin Wang, Gang-Neng Sung, Ming-Kai Chang, and Ying-Yu Shen, “Energy-Efficient Double-Edge Triggered Flip-Flop Design," IEEE Asia Pacific Conference on Circuits and Systems, APCCAS 2006. 4-7 Dec. 2006, pp. 1791 - 1794.

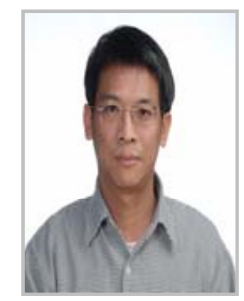

Jih-Fu Tu received his B. S. degree and M. S. degree in Industrial Education from National Kaohsiung Normal University and National Taiwan Normal University, Taiwan in 1983 and 1990 respectively. In 2003, he received the $\mathrm{Ph}$. D. degree in Computer Engineering from Proston University in USA. He is now an Associate Professor in Department of Electronic Engineering, St. John's University from 1990. It interests in the field of computer architectures, integrated circuit design, SoC, intelligential node, and discrete event system for Petri net. 